\title{
The Cost of Erasing Information
}

\author{
J.A. Vaccaro* and S.M. Barnett ${ }^{\dagger}$ \\ ${ }^{*}$ Centre for Quantum Dynamics, Griffith University, Brisbane, Queensland 4111 Australia \\ ${ }^{\dagger}$ Department of Physics, University of Strathclyde, Glasgow G4 ONG, United Kingdom
}

\begin{abstract}
Landauer has argued that the process of erasing 1 bit of information requires a minimum amount of work equal to $k T \ln (2)$ where $T$ is the temperature of a thermal reservoir that consumes the entropy of the bit. We show that, in principle, the thermal reservoir can be replaced by a reservoir of $N$ spin-1/2 particles which is subject to the conservation of spin angular momentum. The cost of erasing 1 bit of information in this case is found to be at least $\beta^{-1} \ln (2)$ where $e^{-\hbar \beta}=\left(N \hbar+2\left\langle J_{z}\right\rangle\right) /\left(N \hbar-2\left\langle J_{z}\right\rangle\right)$ and $\left\langle J_{z}\right\rangle$ is the $z$ component of the total spin angular momentum of the spin reservoir.
\end{abstract}

Keywords: information erasure, thermodynamics, canonical ensemble, spin system PACS: 03.65.Ta, 89.70.-a, 05.70.-a

\section{INTRODUCTION}

Entropy has different incarnations in thermodynamics and information theory. For example, one of the essential elements of thermodynamics is the conservation of energy. Indeed without energy conservation, the maximization of the entropy would lead to the trivial situation of completely disordered systems. In contrast, the Shannon entropy associated with information is independent of energy. In fact, almost all processing of quantum information could take place within a manifold of degenerate eigenstates of energy. As classical information is a special case of quantum information, this energy independence also applies to classical case. The only exception to the energy independence appears to be the processing involved in the erasure of information. Landauer's erasure principle [1] states that the erasure of information incurs an energy cost. Here we show that the physical cost of the erasure of information is directly related to the type of statistical ensemble that is used to absorb the entropy of the unwanted information. In particular, the energy cost in Landauer's erasure principle is incurred because of the use of a thermal reservoir. We show that the thermal reservoir can be replaced by a statistical ensemble of energy degenerate spin- $\frac{1}{2}$ particles. With this replacement the erasure of information incurs a physical cost in terms of spin angular momentum only.

\section{ERASURE MODEL WITH ENERGY COST}

First we describe an erasure model based on a thermal reservoir which incurs an energy cost in accord with Landauer's erasure principle. Let a qubit with a degenerate energy spectrum be in the state of maximum entropy $(|0\rangle\langle 0|+| 1\rangle\langle 1|) / 2$ where $|0\rangle$ and $|1\rangle$ represent states in the computational basis. The qubit represents a 1 bit memory element. In order to reset the qubit state to the logical state $|0\rangle$, it is placed in contact with a 
thermal reservoir at temperature $T$. Since the qubit states are degenerate the qubit is already in thermal equilibrium with the reservoir. Next a splitting is slowly induced in the energy spectrum of the qubit such that the energy of logical state $|1\rangle$ is $E$ higher than $|0\rangle$. This splitting is induced sufficiently slowly that the qubit can always be regarded as being in equilibrium with the thermal reservoir. The state of the qubit when the energy splitting is $E$ is given by the density operator

$$
\frac{|0\rangle\left\langle 0\left|+e^{-E / k T}\right| 1\right\rangle\langle 1|}{1+e^{-E / k T}}
$$

in accordance with the Boltzmann distribution. The work $d W$ required to increase the splitting from $E$ to $E+d E$ while in contact with the reservoir is given by the probability of occupation of the state $|1\rangle$ times $d E$, i.e. $d W=e^{-E / k T}\left(1+e^{-E / k T}\right)^{-1} d E$. The total work needed to increase the splitting slowly from zero to infinity in this way is $W=\int d W=k T \ln (2)$. At the end of this process the state of the qubit is the $|0\rangle$ as required. The qubit is removed from the reservoir and the energy degeneracy is restored at no cost in energy. The erasure here is given by maximizing the entropy subject to the conservation of energy while increasing the energy splitting of the memory qubit.

\section{ERASURE MODEL WITH SPIN COST}

We now consider a reservoir consisting of a collection of $N$ spin- $\frac{1}{2}$ particles which are in thermal equilibrium with a heat bath. The spatial degrees of freedom of the particles have a probability distribution which depends on the temperature of the heat bath and the confining potential. We assume that the Hamiltonian of the system has no spindependent term, and so the probability distributions of the spatial and spin degrees of freedom are decoupled. In this case the thermal motion can be taken to induce random spin exchanges in the particles through collisions. We do not need to consider the heat bath nor the spatial degrees of freedom further. We shall label the eigenstates of the $z$ component of spin angular momentum of each particle as the logical states $|0\rangle$ and $|1\rangle$.

The state space of the spin reservoir can be described as follows. Consider the set of eigenstates corresponding to the eigenvalue $(n-N / 2) \hbar$ of the $z$ component of the spin angular momentum operator $J_{z}$. There are $\left(\begin{array}{l}N \\ n\end{array}\right)$ such states, where $(:)$ is the usual binomial coefficient. We label each element of this set as the collective state $|n, \lambda\rangle\rangle$ where $\lambda=1,2, \cdots,\left(\begin{array}{l}N \\ n\end{array}\right)$ uniquely identifies the state within the set. As an example, the collective state $|N, 1\rangle\rangle$ corresponds to the product of $N$ single particle states $|1\rangle|1\rangle \cdots$. The full basis set of the spin reservoir is given by $\left.\left\{\left|n, \lambda_{n}\right\rangle\right\rangle: n=0, \ldots, N ; \lambda_{n}=1, \ldots,\left(\begin{array}{l}N \\ n\end{array}\right)\right\}$.

In order to model the equilibrium state of the spin reservoir, we treat it as a canonical ensemble but instead of energy being exchanged between it and a larger bath, the two systems randomly exchange the $z$ component of spin. For this we require the bath to also consist of spin- $\frac{1}{2}$ particles. We shall refer to this exchange interaction as "spin-exchange contact". The spin bath maintains the spin reservoir in a state such that the $z$ component of the total spin in the reservoir is given by

$$
\left\langle J_{z}\right\rangle=\left(\alpha-\frac{1}{2}\right) N \hbar
$$


for $0 \leq \alpha \leq 1$. At equilibrium, the probability $P_{n, \lambda}$ of finding the spin reservoir in the state $|n, \lambda\rangle\rangle$ is given by maximizing the entropy $-\sum_{n, \lambda} P_{n, \lambda} \ln P_{n, \lambda}$ with respect to $P_{n, \lambda}$ subject to the constraints $\sum_{n, \lambda} n P_{n, \lambda}=\alpha N$ and $\sum_{n, \lambda} P_{n, \lambda}=1$. The optimum probability distribution is found to be

$$
P_{n, \lambda}=\frac{e^{-n \hbar \beta}}{\left(1+e^{-\hbar \beta}\right)^{N}}
$$

where $\beta=\frac{1}{\hbar} \ln \left(\frac{1-\alpha}{\alpha}\right)$ or, on using Eq. (2), where

$$
e^{-\hbar \beta}=\frac{N \hbar+2\left\langle J_{z}\right\rangle}{N \hbar-2\left\langle J_{z}\right\rangle}
$$

Now imagine another spin- $\frac{1}{2}$ particle is our binary memory element. It begins in the maximally mixed state $\frac{1}{2}(|0\rangle\langle 0|+| 1\rangle\langle 1|)$ and we wish to erase its "memory" and leave it in the logical zero state $|0\rangle\langle 0|$. Let there be a large collection of ancillary spin- $\frac{1}{2}$ particles in the state $|0\rangle\langle 0|$ for our use. The first stage of the erasure protocol entails putting the memory spin in spin-exchange contact with the spin reservoir, letting the combined reservoir-memory spin system come to equilibrium, and then separating the memory spin from the spin reservoir. At this point the state of the memory spin would be

$$
p_{0}|0\rangle\left\langle 0\left|+p_{1}\right| 1\right\rangle\langle 1|
$$

with $p_{1}=e^{-\hbar \beta} /\left(1+e^{-\hbar \beta}\right)=1-p_{0}$. We have assumed that the value of $\left\langle J_{z}\right\rangle=(\alpha-$ $\left.\frac{1}{2}\right) N \hbar$ is maintained by the spin bath and so the value of $\beta$ remains fixed despite the contact with the memory spin. A controlled-not (CNOT) operation is them performed on the memory spin and one ancilla spin, with the former being the control qubit; this yields the state $p_{0}|00\rangle\left\langle 00\left|+p_{1}\right| 11\right\rangle\langle 11|$ where $|x y\rangle$ represents the state $|x\rangle$ of the memory spin and $|y\rangle$ of the ancilla spin. The spin cost of this operation is $\hbar e^{-\hbar \beta} /\left(1+e^{-\hbar \beta}\right)$. The memory-ancilla system is placed in random spin-exchange contact with the spin reservoir while the reservoir-bath is undergoing random spin exchange as before. The spin exchange between the reservoir and the memory-ancilla system is constructed to leave all states unchanged except for the following mapping

$$
|2,1\rangle\rangle|00\rangle \leftrightarrow|0,1\rangle\rangle|11\rangle
$$

where $|n, \lambda\rangle\rangle|i j\rangle$ represents the reservoir collective state $|n, \lambda\rangle\rangle$ and memory-ancilla system state $|i j\rangle$. The random spin exchange continues for a sufficient time for the reservoir and memory-ancilla system to equilibrate. The state of the memory-ancilla spin system is then given by

$$
p_{0}^{\prime}|00\rangle\left\langle 00\left|+p_{1}^{\prime}\right| 11\right\rangle\langle 11|
$$

where now $p_{1}^{\prime}=e^{-2 \hbar \beta} /\left(1+e^{-2 \hbar \beta}\right)=1-p_{0}^{\prime}$. Another ancilla spin is added and a CNOT operation is performed as before to yield the state $p_{0}^{\prime}|000\rangle\left\langle 000\left|+p_{1}^{\prime}\right| 111\right\rangle\langle 111|$ with a spin cost of $\hbar e^{-2 \hbar \beta} /\left(1+e^{-2 \hbar \beta}\right)$. The combined memory-ancilla spin system put in spinexchange contact with the reservoir with the mapping

$$
|3,1\rangle\rangle|000\rangle \leftrightarrow|0,1\rangle\rangle|111\rangle
$$


and so on. After $n$ cycles of this process, the memory-ancilla spins are all in either the logical zero state or all in the logical 1 state with probabilities $p_{0}^{\prime \prime}$ and $p_{1}^{\prime \prime}$, respectively, where $p_{1}^{\prime \prime}=e^{-n \hbar \beta} /\left(1+e^{-n \hbar \beta}\right)=1-p_{0}^{\prime \prime}$. In the limit of many repetitions and large $N$, the memory-ancilla spin system approaches a pure state where each spin is in the logical zero state.

The spin cost of the $n$th cycle is $\hbar$ times the probability $p_{1}^{\prime \prime}$, that is, $\hbar e^{-n \hbar \beta} /\left(1+e^{-n \hbar \beta}\right)$. The total spin cost of the whole process is therefore given by

$$
\Delta J_{z}=\sum_{n=1}^{\infty} \hbar \frac{e^{-n \hbar \beta}}{1+e^{-n \hbar \beta}} .
$$

This sum is approximated by

$$
\beta^{-1} \ln \left(1+e^{-\hbar \beta}\right)<\Delta J_{z}<\beta^{-1} \ln (2) .
$$

If we include the spin of the initial state, then the cost is

$$
\begin{gathered}
\Delta J_{z}^{\prime}=\sum_{n=0}^{\infty} \frac{e^{-n \hbar \beta}}{1+e^{-n \hbar \beta}}=\Delta J_{z}+\frac{1}{2} \hbar, \\
\beta^{-1} \ln (2)<\Delta J_{z}^{\prime}<\beta^{-1} \ln \left(1+e^{\hbar \beta}\right) .
\end{gathered}
$$

Noting that $\beta$ has the same units as $\hbar$, we see that the physical cost of erasure is in terms of spin angular momentum.

\section{CONCLUSION}

Our analysis shows that the physical cost associated with the erasure of information depends on the physical nature of statistical reservoir that is used to absorb the entropy of the memory bit. This cost need not be in terms of energy. We demonstrated this using an example of an erasure protocol based on a statistical ensemble of spin- $\frac{1}{2}$ particles. While the operations needed for the protocol are expected to be difficult to realize in practice, they are nevertheless possible in principle. The cost of the erasure in this case is shown to be in terms of spin angular momentum.

\section{REFERENCES}

1. R. Landauer, IBM J. Res. Lett., 67661 (1961). 